\title{
Gibt es Evidenz zum Nutzen von Evidence-based Medicine?
}

\author{
A. Marx ${ }^{a}$, H. C. Bucher ${ }^{a}$, R. Kunz ${ }^{b}$, G. Antes $^{b}$
}

Was ist eine methodisch gute randomisierte kontrollierte Studie, und wie lassen sich die Ergebnisse von experimentellen Studien in die konkrete Praxis umsetzen? Evidence-based Medicine hat das Rüstzeug entwickelt, um Klinikern und Praktikern mit Hilfe des kritischen Methodikstudiums Antworten auf solche Fragen zu geben. Die anfängliche Aufregung um Evidence-based Medicine («Kochbuchmedizin», Rationierungsinstrument usw.) hat sich gelegt [1]. Evidence-based Medicine beinhaltet keinen Paradigmenwechsel in der Medizin. Evidence-based Medicine hat hingegen die Inhalte der klinischen Epidemiologie für die Beurteilung der wissenschaftlichen Stichhaltigkeit von Beobachtungen und Forschungsergebnissen in der klinischen Medizin neu definiert [2]. In diesem Sinne wird Evidence-based Medicine immer häufiger als Denkschulung für den klinisch tätigen Arzt wahrgenommen. Sie ist ein Instrument, um sich mittels kritischer Beurteilung der Literatur (critical appraisal) ein eigenes Bild zu verschaffen und die klinische Entscheidungsfindung zu optimieren. Neuerdings gewinnt Evidencebased Medicine auch in der Gestaltung der ArztPatient-Beziehung (shared decision making) an Bedeutung [3].

Ziel von Evidence-based Medicine ist der gewissenhafte und vernünftige Gebrauch der besten verfügbaren wissenschaftlichen Evidenz für Entscheidungen in der medizinischen Versorgung. Evidence-based Medicine integriert diese Evidenz und klinische Erfahrung in die individuelle Patientenbetreuung [2]. Die entscheidende Frage, inwiefern Evidence-based Medicine die ärztliche Praxis verändert, bleibt jedoch noch offen. Dass Kurse in Evidence-based Medicine die Fähigkeit zum kritischen Literaturstudium und das klinisch epidemiologische Wissen verbessern, ist in einer kürzlich im «British Medical Journal» publizierten Arbeit nun belegt [4]. Die Autoren zeigen, wie im Rahmen eines 3tägigen Intensivkurses, der auf dem McMaster-Modell des problemorientierten Lernens in Kleingruppen basiert, die Grundthemen der Evidence-based Medicine vermittelt werden. Das Institut für klinische Epidemiologie an den Universitätskliniken Basel bietet gemeinsam mit dem Deutschen Cochrane-Zentrum und dem Institut für Sozial- und Präventivmedizin der Universität Bern erstmals in der Schweiz einen Evidence-based-Medicine-Kurs an, der genau nach diesem erfolgreichen Konzept aufgebaut ist. Der Kurs ist von der Schweizerischen Gesellschaft für Allgemeinmedizin und der Schweizerischen Gesellschaft für Innere Medizin akkreditiert (21 Punkte).

Im Zentrum des Kurses steht die Vermittlung der Grundfertigkeiten zur Beurteilung von wissenschaftlichen Publikationen zu den Themen Diagnose, Therapie, Prognose und Meta-Analyse. Die Erarbeitung des Stoffes erfolgt in einem mehrstufigen Schema:

1. Problem eingrenzen und Formulieren der Frage;

2. gezielte Literatursuche;

3. kritische Bewertung der Evidenz (critical appraisal);

4. Prüfung der Anwendbarkeit der gefundenen Evidenz am konkreten Patienten;

5. Überprüfung des eigenen ärztlichen Handelns.

Der Kurs bietet zusätzlich praktische Übungen zur Literatursuche. Ein Schwergewicht des Kurses wird auf die Schulung der Fähigkeit zum Erkennen und Einschätzen von methodischen Stärken und Schwächen von Studien gelegt. Kommen wir deshalb auf die eingangs gestellte Frage zurück: Was ist eine methodisch gute randomisierte kontrollierte Studie und wie lassen sich die Ergebnisse von experimentellen Studien in die konkrete Praxis umsetzen?

Die jüngste Kontroverse um Sinn und Nutzen des Mammographiescreenings zeigt, dass selbst Experten in der Beurteilung der Qualität von randomisierten kontrollierten Studien zu völlig unterschiedlichen Schlussfolgerungen kommen können. In der Meta-Analyse der «Cochrane Collaboration» wurden nur 2 randomisierte kontrollierte Studien, deren Ergebnisse nicht schlüssig waren, als methodisch verlässlich beurteilt [5]. In einer Meta-Analyse der «US Preventive Services Task Force» wurden hingegen 7 Studien berücksichtigt und eine 20\%ige (95\% Konfidenzintervall 11-29\%) Reduktion der Mammakarzinomsterblichkeit durch das Mammographiescreening dokumentiert [6]. Wie 
Der 2. Oberrheinische EbM-Kurs - Grundlagen und praktische Anwendung, findet vom 27. bis 30. März 2003 in Basel statt.

Information und Anmeldung: Jacqueline D. M. Canonica, Institut für klinische Epidemiologie, Kantonsspital Basel, Universitätskliniken, 4031 Basel, Tel. 061 26531 00, Fax 06126531 09, E-Mail: ebm-kurs@bice.ch, Internet: www.bice.ch
Medicine-Kurse den Teilnehmerinnen und Teilnehmern nebst Know-how Mut zu kritischen Fragen und zum Umgang mit Unsicherheit bringen, ist ein wesentliches Ziel von Evidence-based Medicine erreicht.

\section{Literatur}

1 Sackett DL, Rosenberg WM, Gray JA, Haynes RB, Richardson WS. Evidence-based medicine: what it is and what it isn't. Br Med J 1996; 312(7023):71-2

2 Sackett DL, Richardson WS, Rosenberg W. Evidence-based Medicine. $2^{\text {nd }}$ ed. Churchill Livingstone; 2002.

3 Edwards A, Elwyn G (eds.). Evidence-based Patient Choice. Oxford: Oxford University Press; 2001.

4 Fritsche L, Greenhalgh T, Falck-Ytter Y, Neumayer HH, Kunz R. Do short courses in evidence-based medicine improve knowledge and skills? Validation of Berlin questionnaire and before and after study of courses in evidencebased medicine. Br Med J 2002;325:1338-41.

5 Olsen O, Gøtzsche PC. Cochrane review on screening for breast cancer with mammography. Lancet 2001;358:1340-2.

6 Humphrey LL, Helfand M, Chan BK, Woolf SH. Breast cancer screening: a summary of the evidence for the U.S. Preventive Services Task Force. Ann Intern Med 2002;137:347-60.

7 Goodman SN. The mammography dilemma: a crisis for Evidence-based Medicine? Ann Intern Med 2002;137:363-5.

8 Schulz KF, Chalmers I, Grimes DA, Altman DG. Assessing the quality of randomization from reports of controlled trials published in obstetrics and gynecology. JAMA 1995;272:125-8.

9 Elmore JG, Barton MB, Moceri VM, Polk S, Arena PJ, Fletcher SW. Ten-year risk of false positive screening mammograms and clinical breast examinations. N Engl J Med 1998;338: 1089-96.

10 Rossouw JE, Anderson GL, Prentice RL LaCroix AZ, Kooperberg C, Stefanick ML et al. Risks and benefits of estrogen plus progestin in healthy postmenopausal women: principal results From the Women's Health Initiative randomized controlled trial. JAMA 2002;288(3):321-33.

11 Laine C. Postmenopausal hormone replacement therapy: how could we have been so wrong? Ann Intern Med 2002;137(4):290.

12 Bucher HC, Guyatt GH, Cook DJ, Holbrook A, McAlister FA. Users' guides to the medical literature: XIX. Applying clinical trial results. A. How to use an article measuring the effect of an intervention on surrogate end points. Evidence-based Medicine Working Group. JAMA 1999;282:771-8.

13 Petitti DB. Hormone replacement therapy and heart disease prevention: experimentation trumps observation. JAMA 1998;28:650-2.
Praktikern. Der 2. Oberrheinische EbM-Kurs folgt diesem Grundsatz. Wenn Evidence-based- 